\title{
RFID Model for Simulating Framed Slotted ALOHA Based Anti-Collision Protocol for Muti-Tag Identification
}

\author{
Zornitza Prodanoff ${ }^{1}$ and Seungnam Kang 2 \\ 1 University of North Florida \\ ${ }^{2}$ National Seoul University \\ ${ }^{1}$ USA \\ ${ }^{2}$ South Korea
}

\section{Introduction}

Radio Frequency Identification (RFID) networks use radio signal broadcast to automatically identify items with attached RFID tags. A tag consists of a microchip that stores a unique identifier and an antenna. The tag's antenna is attached to the chip and can transmit a unique tag identifier to a reader (also called interrogator). The reader is capable of learning the set of tags within its interrogation range. The process of learning in-range tags is called a census. After an initial census is completed, the reader can answer queries about the presence of specific tag(s) within its range sent to it from other type of devices.

RFID systems have abundant benefits as compared to the barcode and smart card systems. RFID networks use radio frequency as a method of data transmission. Thus, unlike barcode labels, a tag does not need to be placed in a line of sight position from the reader, or even get in contact with a reader as smart cards, in order to be identified successfully. Depending on whether they use low, high, or ultrahigh transmission frequencies, RFID tags are identifiable within 3 meters span in case of a typical far-field reader [Want06] or at even further distances. Therefore, RFID tags are used more flexibly and conveniently than existing barcode and smart card implementations.

Moreover, some commercial implementations of RFID tags can store data in the amount of 16bytes - 64Kbytes [Finkenzeller03]. RFID tags can hold the same amount of data compared to smart cards, and much larger volume than barcodes. In addition, RFID tags are getting less expensive. The cost of RFID chips at the time of this study is less than 10 cents, while back in 1999, for example, was around 2 US dollars. Since tag readers have limits on their operations range imposed by the frequency of the wireless signal used, when RFID networks need to cover large spaces, multiple readers need to be used. The cost of current reader implementations is hundreds of US dollars. As a result, RFID networks may not be yet suitable to track large inventories of inexpensive items, but they are certainly becoming more affordable and can be used to track different types of items, e.g. live stock, pets, and valuable goods. Due to these advantages RFID systems are emerging as one of the alternative technologies of our time. 
One of the world biggest supply chains Wal-Mart has required suppliers to implement RFID networks in at least 12 of its 137 distribution centres by the end of 2006. The Proctor \& Gamble Co. is the first of about 100 suppliers to conform to Wal-Mart's requirements to tag its products with RFID chips [Computerworld07]. The US Navy finished its pilot of a passive RFID system to support the loading of supplies into cargo containers in May 2004. According to the related final report the RFID process increased the speed and efficiency of the cargo checking process, while less people were needed to support the new RFID based system as compared to the legacy implementation [Weinstein05].

\subsection{Physical composition}

An RFID system is made up of an application, a reader and tags.

- The application is a program installed on a (proxy) computer which can control readers.

- The reader is a device which runs functions such as reading, writing and authentication. When the reader gathers data from tags it transmits to the computer application.

- The tag is used to identify an object and is located on (or in) the object itself.

A reader is connected to the computer and has a transmitter and receiver, while a tag has a control unit (chip) and a coupling element (antenna).

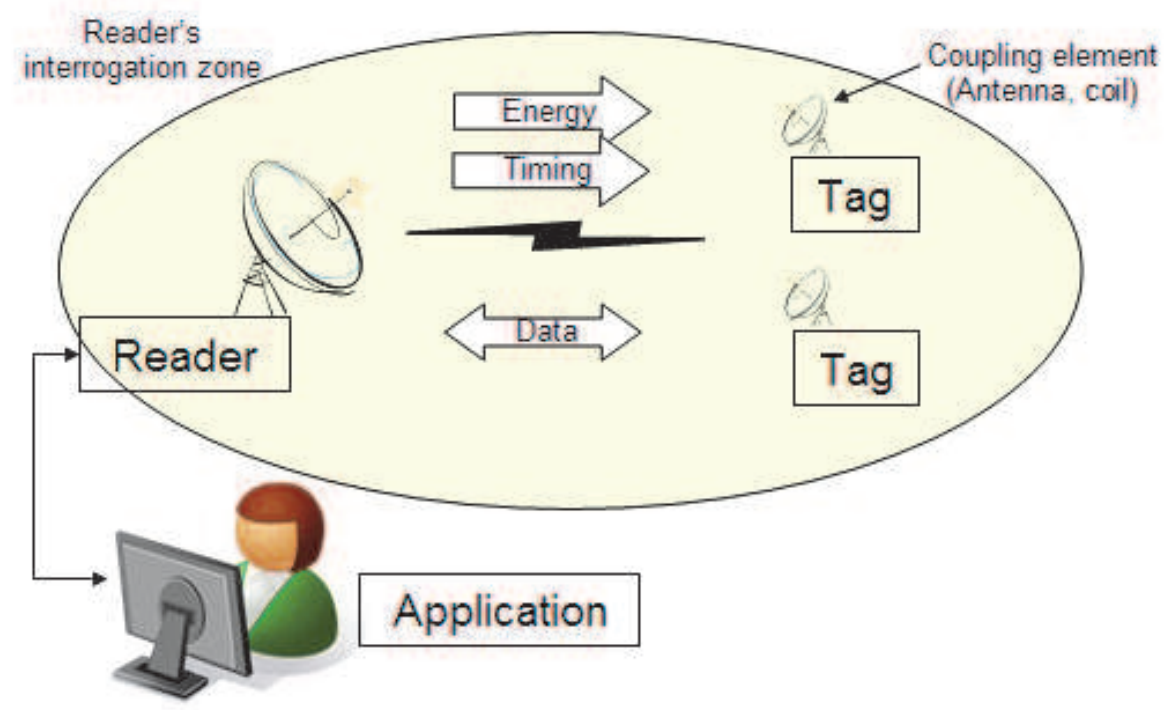

Fig. 1. RFID Physical Composition [Finkenzeller03]

RFID tags can be passive, i.e. not having an internal energy source or active, internal battery powered. A reader typically charges a set of passive tags within its interrogation zone using inductive coupling; the reader broadcasts electromagnetic signal then the tag's antenna absorbs and stores the signal's energy into an on-board capacitor. This technique is called load modulation for near-field coupling and back scattering for far-field coupling. After charging its battery it can be activated. 


\subsection{Framed slotted ALOHA anti-collision algorithm}

The ALOHA algorithm is a collision resolution algorithm based on Time Division Multiple Access (TDMA). There are three flavors of the original ALOHA algorithm: (Pure) ALOHA, Slotted ALOHA and Frame Slotted ALOHA [Zürich04].

In Figure 2, $\mathrm{X}$ and $\mathrm{Y}$ axis represents the read cycle and tags respectively. The read cycle is the time interval between neighboring two REQUEST commands and it can be repeated until all tags in the interrogation range are identified. Note that there no slots are used in the (Pure) ALOHA algorithm (Figure 2: (a)) while the read cycle is divided into several continuous slots in the Slotted ALOHA (Figure 2: (b)) and Framed Slotted ALOHA algorithm. Furthermore, a frame is comprised of the number of slots in the Framed Slotted ALOHA algorithm (Figure 3: A slot is a discrete time intervals synchronized by the reader, sufficiently long in duration to allow a tag to transmit its ID and the ID's 16-bit CRC code. A set of slots are grouped into frames. When size is fixed, each consecutively transmitted frame has the same number of slots.

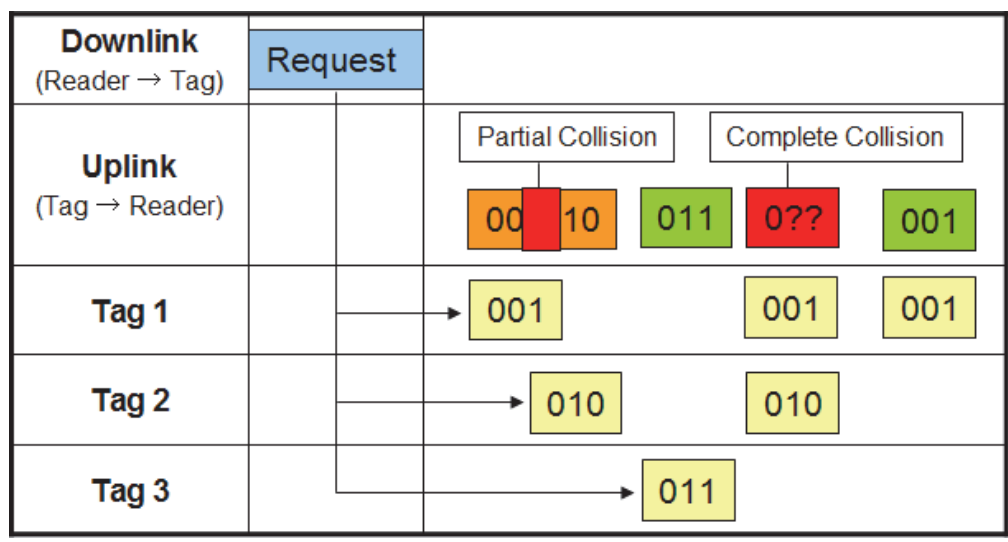

(a) (Pure) ALOHA

\begin{tabular}{|c|c|c|c|c|c|c|c|c|c|c|c|}
\hline $\begin{array}{c}\text { Downlink } \\
\text { (Reader } \rightarrow \text { Tag) }\end{array}$ & Request & & & & & & & Request & & & \\
\hline \multirow{2}{*}{$\begin{array}{c}\text { Uplink } \\
(\text { Tag } \rightarrow \text { Reader })\end{array}$} & & \multicolumn{4}{|c|}{ Complete Collision only } & & & & & \multicolumn{2}{|c|}{ Empty slot } \\
\hline & & $01 ?$ & 001 & ??? & 010 & ??? & ??? & & ??1 & 100 & † \\
\hline Tag 1 & & & 001 & & & 001 & & & & & \\
\hline Tag 2 & & 010 & & & 010 & & 010 & & & & \\
\hline Tag 3 & & 011 & & 011 & & 011 & & & 011 & & \\
\hline Tag 4 & & & & 100 & & 100 & & & & 100 & \\
\hline Tag 5 & & & & & & & 101 & & 101 & & \\
\hline
\end{tabular}

(b) Slotted ALOHA

Fig. 2. Pure and Slotted ALOHA Algorithms 
The reader broadcasts the REQUEST command to the tags located in the reader's interrogation range during the downlink while the tags transmit their data to the reader during the uplink. As all activated tags share the uplink partial or complete collision can occur in the (Pure) ALOHA algorithm. However, if the data is transmitted using the slot of frame the partial collision can be eliminated. Furthermore, to reduce the fraction of collision occurrence tags send their data no more than once within a frame, which is the Frame Slotted ALOHA algorithm. We next present in more detail the operation of the three ALOHA algorithms introduced above.

\subsection{1 (Pure) ALOHA}

A tag itself decides the data transmission time randomly as soon as it is activated. The transmission time is not synchronized with both the reader and the other tags at all. When the electricity is charged by the reader's electromagnetic wave tags transmit data after receiving the REQUEST command from the reader. If multiple tags transmit data imminently (whether earlier or later) then a complete or partial collision occurs (Fig. 2 (a)). Retransmitting after random delay is the solution for a collision. During the read cycle the reader receives the data and identifies tags sent data without collision. When a read cycle is done then the reader broadcasts the SELECT command with the tag's unique identifier received from the tag. Once tags are selected the tags stop responding for the request command i.e. the selected tags keep silence until whether they receive other commands e.g. authenticate, read and write or the tag's power is off by being located out of the reader's power range. When the tag is reentered into the reader's interrogation range it restart transmitting its data to the reader. The advantage of this algorithm is simplicity.

\subsubsection{Slotted ALOHA}

It is obtained by the addition of a constraint to the (Pure) ALOHA. The read cycle is divided into discrete time intervals called slot and which is synchronized with the entire tags by the reader. Thus, tags must choose one of the slots randomly and transmit data within a single slot. Transmission begins right after a slot delimiter (Fig. 2 (b)). This causes that packets either collide completely or don't collide at all i.e. there is no partial collision in the Slotted ALOHA algorithm. This reduces wasting the read cycle relatively as compared with the (Pure) ALOHA algorithm. However, the empty slot can be occurred in the read cycle and the disadvantage is that it requires a synchronization mechanism in order for the slot-begin to occur simultaneously at all tags.

\subsubsection{Framed slotted ALOHA}

Framed Slotted ALOHA algorithm uses the frame which is the discrete time interval of the read cycle and each frame is divided into the same number of slots. There are multiple frames in a single read cycle and the frame size is decided by the reader (Figure 3: There is a constraint that the tags can transmit data only once in each frame. It may reduce the number of collided slots and it shows the best performance among them.

\subsection{Classification of the framed slotted ALOHA protocol}

FSA (Framed Slotted Aloha) can be classified into the BFSA (Basic Framed Slotted Aloha) and the DFSA (Dynamic Framed Slotted Aloha) according to whether which uses fixed frame size or variable frame size [Klair04]. If the number of actual tags is unknown DFSA 
can identify tags efficiently rather than BFSA by changing frame size since BFSA uses fixed frame size. In addition, BFSA and DFSA can be further classified based on whether they support muting or/and early-end features [Klair04]. The muting makes tags remain silent after being identified by the reader while the early-end allows a reader close an idle slot early when no response is detected. Figure 4 is shown for the classification of the FSA.

\begin{tabular}{|c|c|c|c|c|c|c|c|c|c|c|c|}
\hline $\begin{array}{c}\text { Downlink } \\
\text { (Reader } \rightarrow \text { Tag) }\end{array}$ & Request & & & Fram & & & & Request & & & \\
\hline Unlink & & Compl & ete Colli & sion only & त & Empty & slot & & & & \\
\hline (Tag $\rightarrow$ Reader) & & 010 & 011 & $? 0 ?$ & $\checkmark$ & 101 & • ( & & & 100 & 001 \\
\hline Tag 1 & & & $\longrightarrow$ & 001 & & & & & & & 001 \\
\hline Tag 2 & & 010 & & & & & & & & & \\
\hline Tag 3 & & & 011 & & & & & & & & \\
\hline Tag 4 & & & & 100 & & & & & & 100 & \\
\hline Tag 5 & & & & & & 101 & & & & & \\
\hline
\end{tabular}

Fig. 3. Framed Slotted ALOHA Algorithms

1) BFSA

- BFSA-Non Muting

- BFSA-Muting

- BFSA-Non muting-early-end

- BFSA-Muting-early-end

2) DFSA

- DFSA-Non Muting

- DFSA-Muting

- DFSA-Non muting-early-end

- DFSA-Muting-early-end

Fig. 4. Classification of FSA

\section{RFID network protocol simulation using OPNET}

Framed Slotted ALOHA and Binary Tree are the two most widely used multi tags identifying anti-collision protocols. Fabio Cappelletti et al. simulated the Binary Tree protocol of RFID by using the OPNET IT Guru 11.0 in 2005 [Cappelletti06]. In the paper, they measured the network throughput and the census delay through the simulation. And they compared simulation performance and analytical results. What they measure is shown in Figure 5. 


\begin{tabular}{|c|c|c|}
\hline Analytical Parameters & Simulation parameters & Unit \\
\hline Network throughput & Network throughput & $(\%)$ \\
\hline Throughput per node & Throughput per node & $(\%)$ \\
\hline $\begin{array}{l}\text { Total census delay } \\
\text { Lower bound } \\
\text { Upper bound } \\
\text { Arithmetic average } \\
\text { Heuristic }\end{array}$ & Total census delay & $\begin{array}{l}\text { Number } \\
\text { of slots }\end{array}$ \\
\hline $\begin{array}{l}\text { Time required to detect a single tag } \\
\text { Lower bound } \\
\text { Upper bound } \\
\text { Arithmetic average } \\
\text { Heuristic }\end{array}$ & Time required to detect a single tag & $\begin{array}{l}\text { Number } \\
\text { of slots }\end{array}$ \\
\hline $\begin{array}{c}\text { Number of transmitted packets } \\
\text { (Total, Average) }\end{array}$ & & $\begin{array}{l}\text { Number } \\
\text { of packets }\end{array}$ \\
\hline
\end{tabular}

Fig. 5. The Measured Parameters for Analysis and Simulation

The network throughput represents the ratio between the number of successfully transmitted packets and the total number of packets sent by the tags while the throughput per node denotes the average number of packets sent by a single tag. The results of the paper showed that the analytical performance was in good agreement with the simulation results.

\subsection{Framed slotted ALOHA protocol performance evaluation using Philips I-Code system}

\subsubsection{The I-Code RFID system}

It is commercial product of RFID system which is comprised of the actual tags and a reader connected to the computer. The application is installed in the computer to control the reader collecting data from tags. The built-in Framed Slotted ALOHA protocol is provided by the system. The memory size of an I-Code tag is the total of 64 bytes which are available for 46 bytes application data, 8 bytes serial number and 10 bytes functionalities such as write protection, maintaining quiet state of tag and reset quite state, etc. And, the reader provides the interface for setting configuration parameters such as the serial connection speed and commands for handling communication with tags. Examples of commands are following:

- Anti-collision/select (ACS): After broadcasting this command, tags begins transmitting their serial numbers. Once tags become "selected" status then remain quite in following ACS commands.

- $\quad$ Read: This command makes the selected tags transmit their data to the reader.

- Write: This command makes the selected tags write data transmitted by the reader.

\subsubsection{The procedure of tag identification}

The procedure of tag identification is different based on the classification of RFID [Finkenzeller03]. There are two types of procedure based on the tags characteristic in the Frame Slotted ALOHA protocol. One of them switches off when read while the other not switches off but replays transmission. The I-Code system uses the switching off tag. Figure 6 depicts the census procedure used in the I-Code System implementation. 


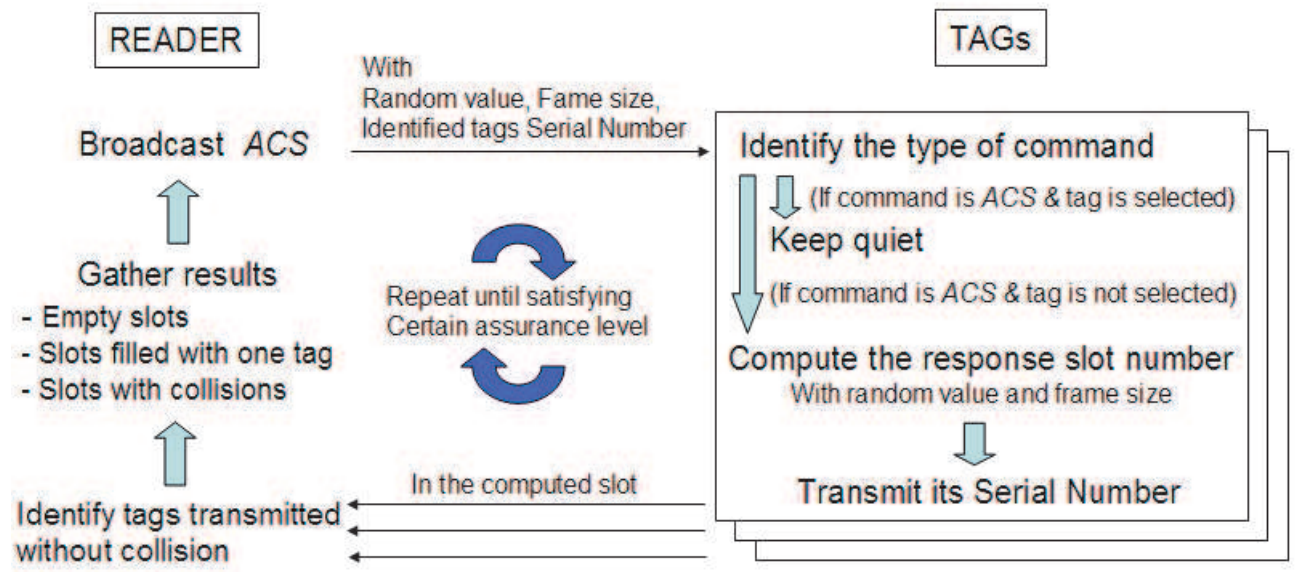

Fig. 6. Tag Identification Procedure of the I-Code System

The reader broadcasts the ACS command with a random value, frame size to make tags transmit their serial number to the reader. When the tag receives a command from the reader first of all it identifies the type of command, and if it is ACS and the status of tag is not 'selected' then it computes the response slot number using the random value and frame size ( $0 \leq$ response slot < frame size) as parameters. Random value is used to prevent the same collisions from occurring repeatedly. The serial number in the computed slot is transmitted to the reader by the tag. During an uplink, data transmission from tags to reader, the reader can identify tags transmitted without collision. The results of a read cycle (the number of empty slots, the number of slots filled with one tag and the number of collided slots) are used for analysis. Once the tag is identified the tag remains quiet until other command e.g. read or write is broadcasted with serial number. And if the tag re-enters the reader's power zone after moving out, that needs re-identification. The read cycle is repeated till reaching the assurance level, the probability of identifying all tags in the reader's range.

\subsubsection{Basic framed slotted ALOHA}

Through simulation we can measure the total census delay by varying the frame size for given number of tags. Then we can find the optimal frame size which results in the minimum total census delay, for given number of tags (see Figure 7).

The optimal frame size, resulting in minimum census delay, can be determined according to the total number of tags. Figure 8 presents an example of the relationship between total number of tags and frame size. For example, the optimal frame size for 80 active tags is 45 , while for 30 passive tags it is 40 .

\subsubsection{Dynamic slot allocation for dynamic framed slotted ALOHA}

To maximize network throughput frame size (the number of allocated slots in the read cycle) should be chosen in accordance with the number of tags since for the same fixed slot size, number of total collisions during a census increases with increase in total number of tags. 


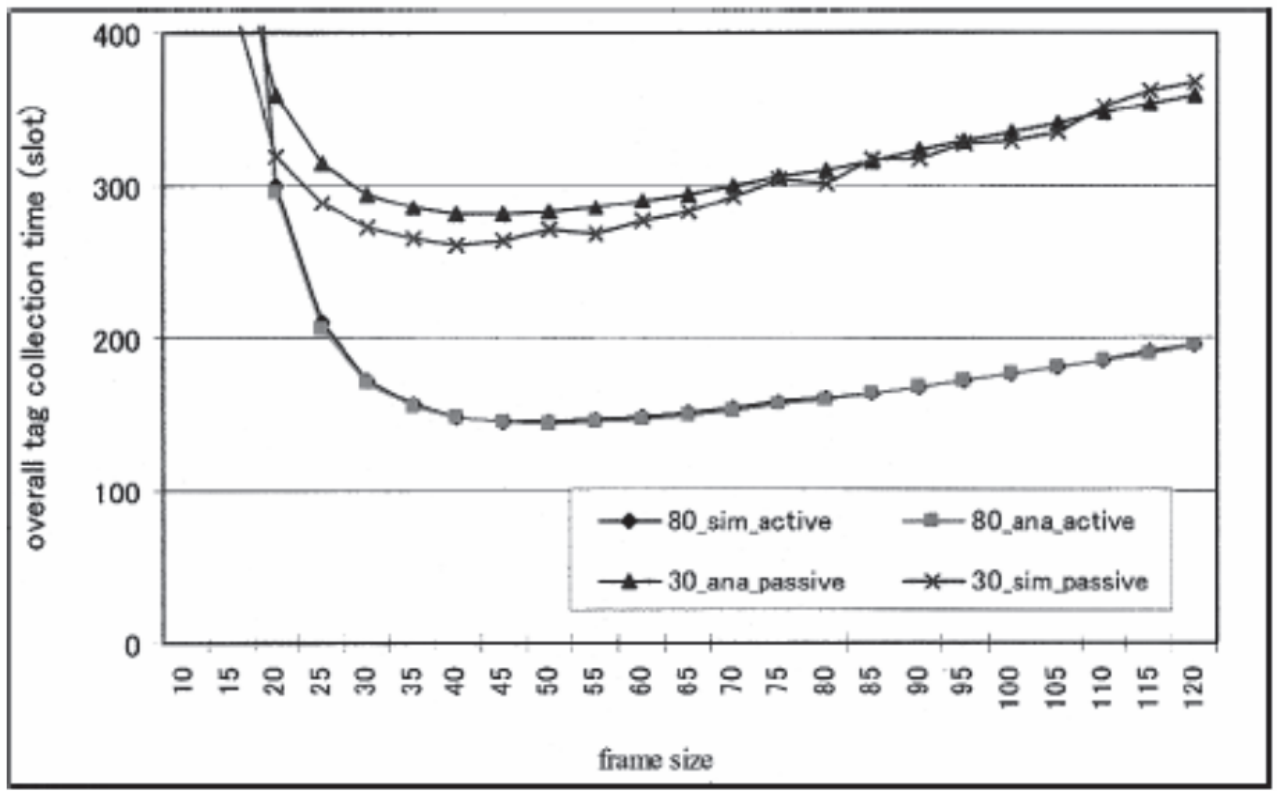

Fig. 7. Example of Total Census Delay (Tag Collection Time) Using Static Frame Size [Bin05]

Due to the nature of the Framed Slotted ALOHA protocol, the read cycle time is divided by the number of slots in a frame and packet data (tag ID number plus CRC) to be transmitted should occupy a single slot. If there are lots of tags and frame size is small then the probability of collisions will be increased and the number of identified tags is decreased, because tags will be competing for a lesser number of slots within a frame. On the contrary, if the reader reads few tags with too big frame size then the probability of collision is decreased, but at the expense of the response time being increased. There is optimal frame size that makes minimum number of read cycles for certain number of tags. The dynamic slot allocation is choosing the optimal frame size (in number of fixed slots) during the tag read cycle. However, the problem is that the number of tags is unknown. So, the reader should estimate the number of tags in each read cycle with the result of the previous read cycle (number of empty slots, number of slots filled with one tag, number of collided slots) and current frame size.

\subsection{Developed approaches of the framed slotted ALOHA protocol}

The key difference of the developed Framed Slotted ALOHA protocol is how they estimate the number of tags and what they estimate.

\subsubsection{H. Vogt's algorithm}

In this scheme, they estimate the number of tags using the current frame size and the result of read cycle. And then updates the current frame size using the estimated number of tags and previous frame size. The procedure of this algorithm is shown in Figure 9. Variable ' $\mathrm{N}$ ', 'N0', 'n_est' and 'stepN' represent the frame size, temporary frame size, the 
estimated number of tags and the counter for the cycle performed with currently estimated framed size, respectively. Variable ' $c$ ', ' $t$ ' represents the result of a read cycle comprised of three integers; number of empty slots, number of slots filled with one tag and number of collided slots, and variable ' $\mathrm{t}$ ' represents the temporary number of estimated number of tags.

In order to estimate the number of tags two estimation functions are used; lower bound and Chebyshev's inequality. Lower bound simply estimates the number of tags is bigger than the summation of the number of slots filled with one tag and two-times of the number of collided slots:

Number of tags $\geq$ Number of filled slots with one tag $+2 \times$ Number of collided slots (1)

When the lower bound is used the real value of number of tags is underestimated.

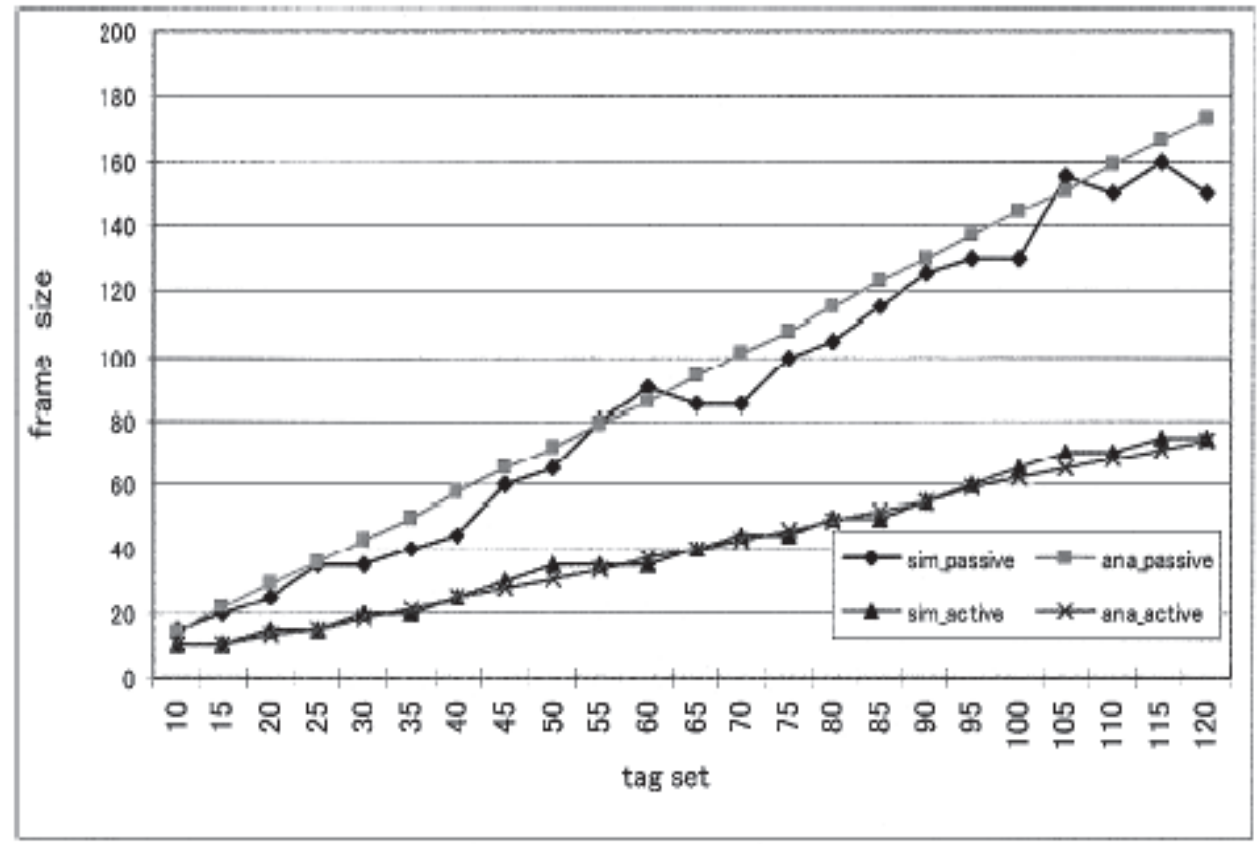

Fig. 8. Example of Optimal Frame Size For Minimum Census Delay [Bin05]

Chebyshev's inequality measures the difference the real values and expected values to estimate the number of tags for which the difference becomes minimal [Vogt02]. The number of tags are estimated using the currently used frame size $(\mathrm{N})$ and the results of previous read cycle $<\mathrm{c}_{0}, \mathrm{c}_{1}, \mathrm{c}_{\mathrm{k}}>$ representing the number of empty slots, the number of slots filled with one tag and the number of collided slots respectively. And $<a_{0}^{N, n}, a_{1}^{N, n}, a_{\geq 2}^{N, n}>$ denotes the expected number of empty slots, the expected number of slots filled with one tag and the expected number of collided slots respectively where $\mathrm{N}$ and $\mathrm{n}$ represent the frame size and the number of tag respectively. Lower bound is more accurate for low values of $\mathrm{n}$ while Chebyshev's inequality is steadier for a wider range of $\mathrm{n}$ [Vogt02]. 


$$
\varepsilon_{v d}\left(N, c_{0}, c_{1}, c_{k}\right)=\min \left|\left(\begin{array}{l}
a_{0}^{N, n} \\
a_{1}^{N, n} \\
a_{\geq 2}^{N, n}
\end{array}\right)-\left(\begin{array}{l}
c_{0} \\
c_{1} \\
c_{k}
\end{array}\right)\right|
$$

* Function: VogtAlgorithm ()

- Variable: integer : N, N0, n_est, stepN, c, t

- $\mathrm{N}=$ minimum frame size

- repeat begin while step $\mathrm{N}$ is smaller than maxStep:

stepN++;

Perform a read cycle with $\mathrm{N}$ and store the result in $\mathrm{c}$

Estimate number of tags with $\mathrm{N}$ and $\mathrm{c}$, and then store result in $\mathrm{t}$

If $t$ is bigger than $n \_$est

then save $t$ in n_est.

Call adaptFrameSize(N, n_est) and save value in N0.

If $\mathrm{N} 0$ is bigger than $\mathrm{N}$

then reset stepN with 0 . And, save N0 in N.

- repeat end

* Function: adaptFrameSize(N, n_est)

- Return type : integer

- repeat begin while $n \_$est is smaller than low value for choice of $\mathrm{N}$ :

Store $\mathrm{N} / 2$ in $\mathrm{N}$.

- repeat end

- repeat begin while $\mathrm{n} \_$est is bigger than high value for choice of $\mathrm{N}$ :

Store $2^{*} \mathrm{~N}$ in $\mathrm{N}$.

- repeat end

Fig. 9. Pseudo Code of H. Vogt's Algorithm

Figure 10 presents the optimal frame size resulting from the execution of function adaptiveFrameSize ( $N$, n_est) using as an input the estimated number of tags and the value of the current frame size $N$. The optimal frame size is selected for a range of tags, based on a low and high margin.

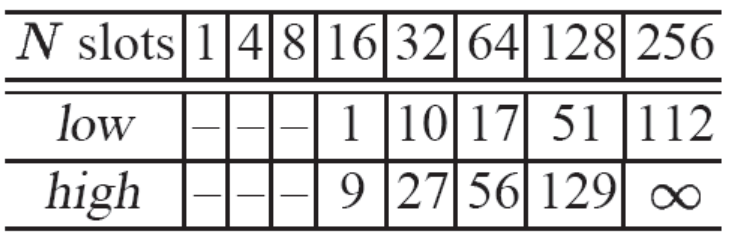

Fig. 10. Choosing Optimal Frame Size [Vogt02]

\subsubsection{Bin ZHEN et al.'s algorithm}

This algorithm estimates the number of tags using the posteriori probability. The posteriori probability of $k$ tags for an observed slot is as below: 


$$
p_{k}^{0}(i)=\left\{\begin{array}{c}
0 \quad \text { if } k=0,1 \\
\text { if } k \geq 2 \\
\frac{p_{k}(i)}{1-p_{0}(i)-p_{1}(i)}
\end{array}\right.
$$

The a posteriori expected value of the number of tags is respectively, 0 for an empty slot, 1 for a slot filled with one tag, and $\sum_{k=2}^{N} k p_{k}^{0}(i)$ tags for a collided slot. Thus, the estimated tag sets from the current read cycle is $p_{1}(i)+\sum_{k=2}^{N} k p_{k}^{0}(i)$. Then, the estimated number of tags comes from the result of the $i$ th read cycle is,

$$
n_{e s t}(i+1)=s+K g
$$

Where $K=2.39$ is a constant for collided slots [Bin05]. And, the frame size $(N)$ in the $(i+1)$ th read cycle is

$$
\begin{aligned}
& N(i+1)=H^{*} n_{\text {est }}(i+1) \\
& \left\{\begin{array}{l}
H=1-1.4 \text { Passive tag } \\
H=0.8-1 \text { Active tag }
\end{array}\right.
\end{aligned}
$$

where $H$ is a constant, which maps the tags to the frame size. The update of the frame size occurs when $n_{\text {est }}(i+1) \geq \gamma{ }^{*} n_{\text {est }}(i)$. Here, $\gamma$ is constant value which is 1.15 and it denotes a threshold to handle random jitter of number of estimated tags. The procedure of this algorithm is shown in Figure 11.

* Function: BinZHENAlgorithm ()

-Variable: integer : $\mathrm{N}, i, \mathrm{c}, \mathrm{s}, \mathrm{n}_{\text {est }}(i)$

$\mathrm{N}=$ minimum frame size

$i=0$

- repeat begin while $i$ is smaller than the number of read cycle for confidence level:

$i++$;

Perform a read cycle with $\mathrm{N}$ and store the result in c for collided slots, $\mathrm{s}$ for success slots.

Estimate number of tags with $\mathrm{N}, \mathrm{c}$ and $\mathrm{s}$, and then store result in $\mathrm{n}_{\mathrm{est}}(i)$.

If $n_{\text {est }}(i)$ is bigger than $\gamma^{*} n_{\text {est }}(i-1)$

then Call calculateFrameSize $\left(\mathrm{n}_{\text {est }}(i)\right.$, tagType $)$ and save value in $\mathrm{N}$.

$$
i=0 \text {. }
$$

- repeat end

* Function: calculateFrameSize( $\mathrm{n}_{\text {est }}(i)$, tagType $)$

- Return type : integer

- If tagType is passive

then $\mathrm{N}(i)=($ random value of $1-1.4) * \mathrm{n}_{\mathrm{est}}(i)$

else $\mathrm{N}(i)=($ random value of $0.8-1){ }^{*} \mathrm{n}_{\text {est }}(i)$

- return $\mathrm{N}(i)$

Fig. 11. Pseudo Code of Bin ZHEN et al.'s Algorithm 


\subsubsection{EDFSA (Enhanced Dynamic Framed Slotted ALOHA)}

This algorithm estimates the number of unread tags instead of number of tags to determine the frame size. H. Vogt's algorithm shows poor performance when the number of tags becomes large because the variance of the tag number estimation is increased according to the number of tags increase [Rom90]. Therefore, to handle the poor performance of large number of tag identification EDFSA algorithm restricts the number of responding tags as much as the frame size. Conversely, if the number of tags is too small as compared with the frame size it reduces the frame size. To estimate the number of unread tags equation (2) is used. The procedure of EDFSA algorithm's read cycle is shown in Figure 12.

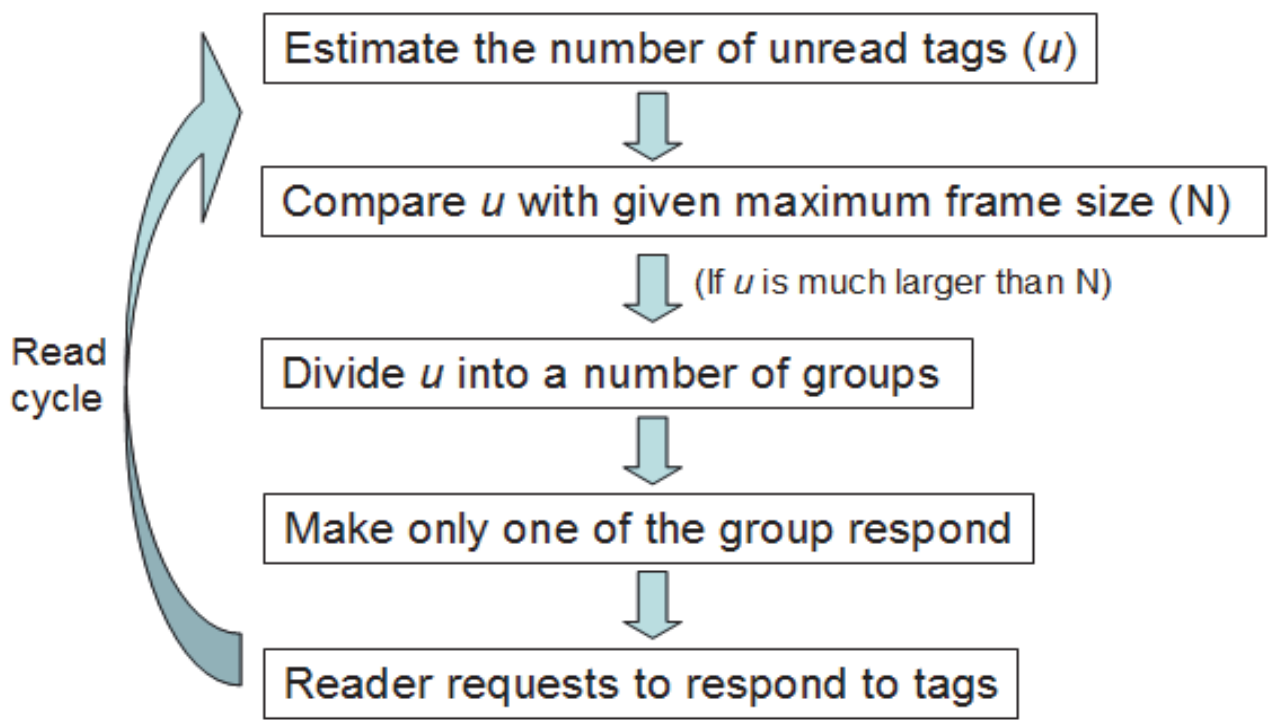

Fig. 12. Read Cycle of EDFSA Algorithm

\subsection{Evaluating delays}

To evaluate the implementation of the BFSA protocol I first evaluated the total census delay of the tag reading process. It is comprised of three different delays; success delay, collision delay and idle delay. Thus, the total census delay is defined as

$$
T[n]=n+C[n]+I[n]
$$

where $n$ is success delay, $C[n]$ is collision delay and $I[n]$ is idle delay [Cappelletti06]. The unit of delay can be defined as a slot duration $T(\mathrm{sec})$ and it is defined as,

$$
T=\frac{\text { ID }(\text { bits })}{\text { data_rate }(\text { bps })}
$$

where ID (bits) is the size of the packet containing tag's ID, and data_rate (bps) is the data rate from tag to reader. 


\subsubsection{BFSA-non-muting}

It is necessary that evaluating of the read cycles satisfying the confidence level a since it is used to determine total census delay. The assurance level $a$ is the probability of identifying all tags in the reader's interrogation range [Vogt02] e.g. if $a=0.99$ which means one or more missing tags, less than $1 \%$ of all, are allowed. The probability of $r$ tags responding in a slot in the $i$ th read cycle is given by [Bin05]

$$
p_{r}(i)=\left(\begin{array}{l}
n \\
r
\end{array}\right)\left(\frac{1}{N}\right)^{r}\left(1-\frac{1}{N}\right)^{n-r}
$$

where $\mathrm{N}$ is the given frame size (slots) and $n$ is the number of tags to be read in the $i$ th read cycle. From the equation 8 , the probability of having one or more idle $\left(p_{o}(i)\right)$, successful $\left(p_{1}(i)\right)$, and collide $\left(p_{k}(i)\right)$ slots in the $i$ th read cycle are defined as:

$$
\begin{gathered}
p_{0}(i)=\left(1-\frac{1}{N}\right)^{n} \\
p_{1}(i)=\frac{n}{N}\left(1-\frac{1}{N}\right)^{n-1} \\
p_{k}(i)=1-p_{1}(i)-p_{0}(i)
\end{gathered}
$$

Then the expected number of the successful transmissions in the $i$ th read cycle becomes $N p_{1}(i)$ since a read cycle has $N$ slots [Bin05]. The probability of having an unread tag after $R$ read cycle is given by [Bin05] [Klair04]

$$
p_{\text {miss }}(i)=\prod_{i=1}^{R}\left(1-\frac{N p_{1}(i)}{n}\right)=1-\alpha
$$

$R$ represents the number of required read cycles to identify a set of tags with a confidence level a. As the number of tags $n$ and the frame size $N$ are the same for all read cycle, $p_{1}(i)$ is constant. That makes equation 13 as,

$$
p_{\text {miss }}(i)=\left(1-\frac{N p_{1}}{n}\right)^{R}=1-\alpha
$$

If we solve the equation 13 for $R$ we can obtain the condition of $R$ as below: [Klair04]

$$
R \geq\left\lceil\frac{\log (1-\alpha)}{\log \left(1-\frac{N p_{1}}{n}\right)}\right\rceil=\left[\frac{\log (1-\alpha)}{\log \left(1-\frac{n\left(1-\frac{1}{N}\right)^{n-1}}{n}\right)}\right]=\left[\frac{\log (1-\alpha)}{\log \left(1-\left(\frac{N-1}{N}\right)^{n-1}\right)}\right]
$$


The ceil function is used since $R$ is the integral value. By using $R$ and if the number of tags is known, we can evaluate the theoretical delay of successful $(n)$, idle $(I[n])$, and collision $(C[n])$ transmission as follows [Klair04]:

$$
\begin{gathered}
n=N p_{1} R T \\
I(n)=N p_{0} R T \\
C(n)=N R T\left(1-p_{0}-p_{1}\right)
\end{gathered}
$$

where $N$ is a frame size, $T$ is slot duration. The summation of those three delays represents the total census delay.

\subsubsection{BFSA muting}

Muting decreases the number of tag's responses after every read cycle. Hence, the number of responding tags in the $(i+1)$ th read cycle is less than or equal to those in the ith read cycle. The number of responding tags in the $(i+1)$ th read cycle is evaluated as [Bin05],

$$
n(i+1)=n(i)-p_{1}(i) \times N
$$

where $p_{1}(i) \times N(i)$ represents the number of tags muted in the ith read cycle. And we can calculate the $R$ with the given $n$ and $N$ by using the equation 14 . Then the collection rounds to read all tags $R$ is given by solving the following equation [Bin05]

$$
p_{\text {miss }}(i)=\prod_{i=1}^{R}\left(1-\frac{N p_{1}(i)}{n(i)}\right)=1-\alpha
$$

By using $R_{\min }$, if the number of tags is known, we can evaluate the theoretical minimum delay of successful $(n)$, idle $(I[n])$, and collision $(C[n])$ transmission by using the equation 15 , 16 , and 17. And, their summation yields the minimum total census delay.

\subsection{Evaluating network throughput}

Network throughput can be defined as the ratio between the number of successfully transmitted packets (one per tag) and the total number of packets sent by the tags during the census [Cappelletti06]. Suppose that there are $n$ tags to be read. Then, the total number of packets sent by $n$ tags during a census for non-muting BFSA is

$$
P[n]=n R
$$

where $R$ is the number of required read cycles needed to identify a set of tags with a confidence level $a$. Since tags can transmit only once in a read cycle. Now we can calculate the network throughput as

$$
S[n]=\frac{\alpha n}{P[n]}=\frac{\alpha}{R}
$$

where $a$ is assurance level, $n$ is total number of identified tags, and $P[n]$ is the total number of packets sent by the tags during the census. 


\subsection{Validation of the models}

In this project, I implemented two Aloha models; BFSA-Muting and BFSA-Non-Muting. To validate the model I analyzed the log file [appendix A, B] of the models and compared with the pseudo code. For easy comparison I put the figures describing the events of the simulation comes from the log file.

\subsubsection{Simulation information}

For the simplicity I put a reader and eight tags, and the same given conditions are used between two simulations. The reader and tags being used in the simulation are shown in Figure 13 (a) while the given conditions are shown in Figure 13 (b).
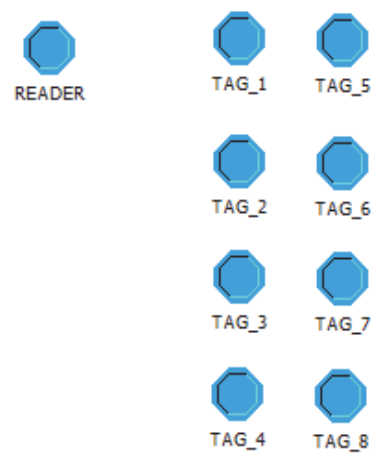

\begin{tabular}{|l|lr|}
\hline \multicolumn{1}{|c|}{ List } & \multicolumn{2}{c|}{ Value } \\
\hline Assurance level & \multicolumn{2}{|c|}{0.99} \\
\hline Data rate (reader $\leftrightarrow$ tag) & 500,000 (bps) \\
\hline Number of slots in a read cycle & 8 & (ea) \\
\hline Duration of a read cycle & 0.00128 (sec) \\
\hline Duration of a single slot & 0.00016 (sec) \\
\hline REQUEST packet size (reader $\rightarrow$ tag) & 88 & (bits) \\
\hline SELECT packet size (reader $\rightarrow$ tag) & 72 & (bits) \\
\hline RESPONSE packet size (tag $\rightarrow$ reader) & 80 & (bits) \\
\hline
\end{tabular}

(a) A Reader and Tags

(b) Given Simulation Conditions

Fig. 13. Simulation Information

The time required for the packet transmission can be calculated by using the given packet size and the data rate among reader and tags. They are shown in Figure 14.

\begin{tabular}{|l|l|}
\hline \multicolumn{1}{|c|}{ List } & Value \\
\hline REQUEST packet & 0.000176 (sec) \\
\hline SELECT packet & 0.000144 (sec) \\
\hline RESPONSE packet & $0.00016 \quad$ (sec) \\
\hline
\end{tabular}

Fig. 14. Packet Transmission Time

I assume that the propagation delay is negligible since in case of a typical far-field reader has 3 meters span interrogation range [Want06]. Consider the speed of light is 299,792,458 $\mathrm{m} / \mathrm{s}$ then the delay of 3 meters will be $1^{-8}$ seconds. And I also assume the calculation delay of the reader and of the tag is negligible as simplicity is the strong point i.e. it does not need complex calculation both for the reader and for the tag.

\subsubsection{BFSA-muting}

For the validation of the simulation model we compared the analytical results (obtained based on an algorithm presented in [Klair04] (see Figure 15)) with our simulation results. 
When the reader starts a census procedure the number of unread tags is initialized to the number of actual tags in range. While the census is performed to identify unread tags the number of identified tags, collided slots, idle slots, and the current frame size are stored as a running total. If there is no collision from tags the total delay, collision delay, and idle delay are calculated. $T$ represents the duration of a single slot.

The log from the BFSA-Muting simulation is shown in Appendix A. Figure 16 depicts the sequence of events during the BFSA-Muting simulation. Through analyzing the log we can check the correctness of the implementation.

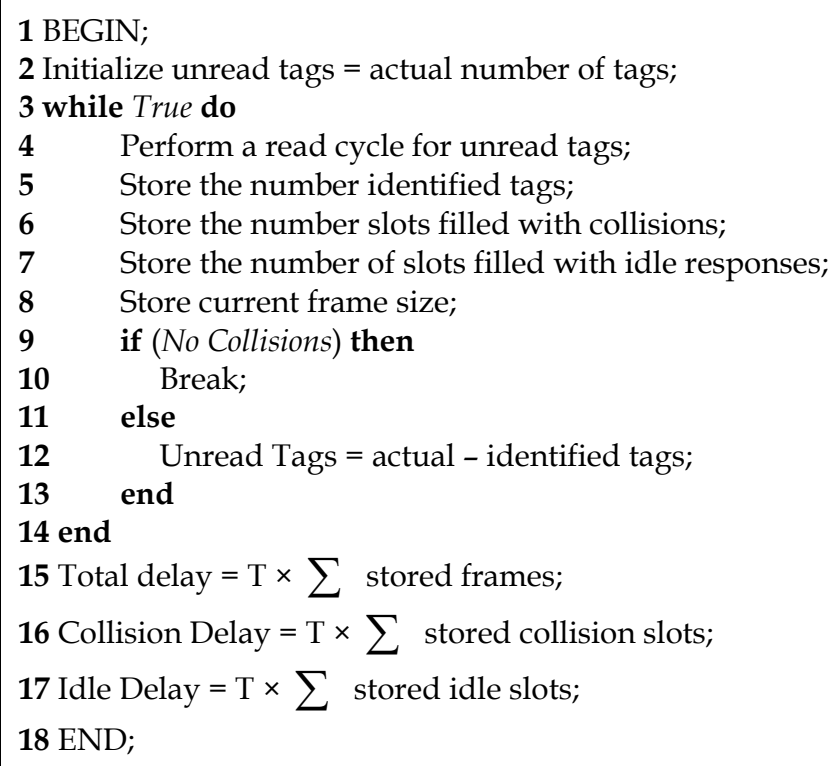

Fig. 15. Pseudo Code of the BFSA Muting

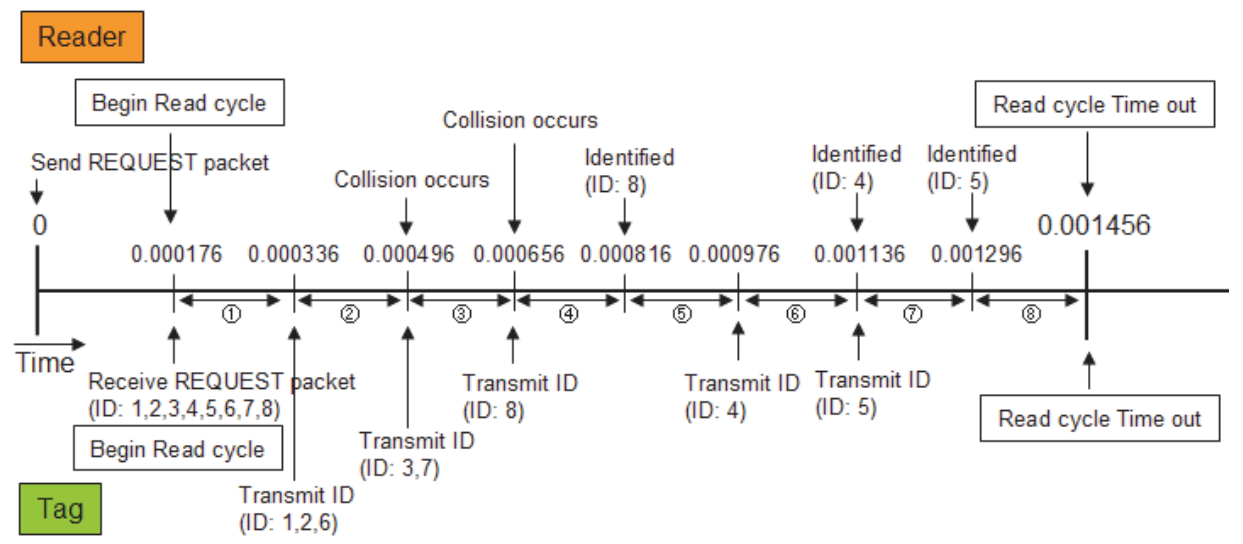

(a) First Read Cycle of the Simulation 


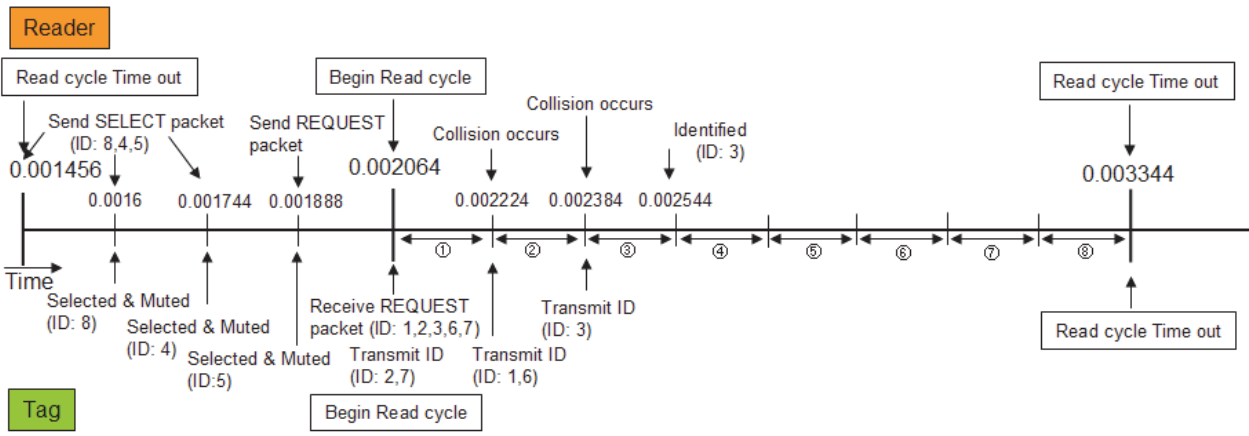

(b) Second Read Cycle of the Simulation

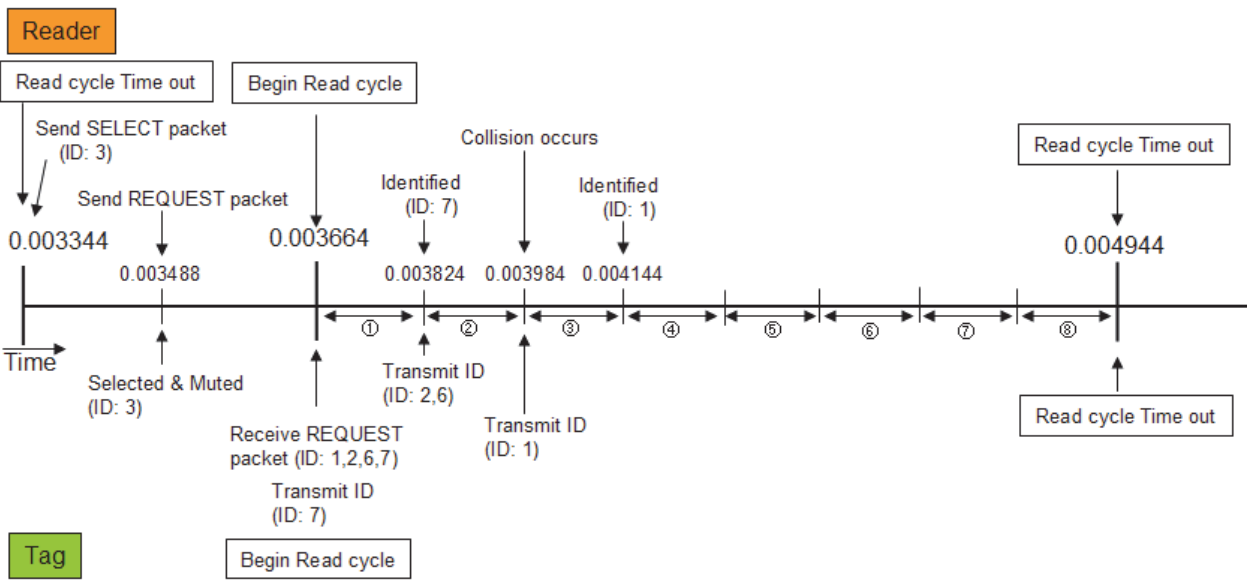

(c) Third Read Cycle of the Simulation

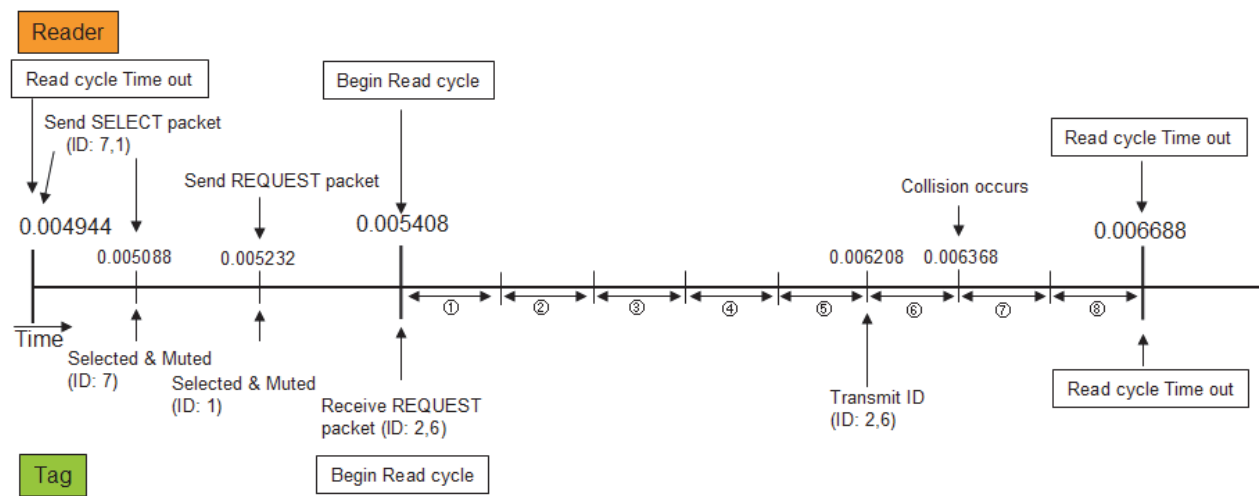

(d) Fourth Read Cycle of the Simulation 


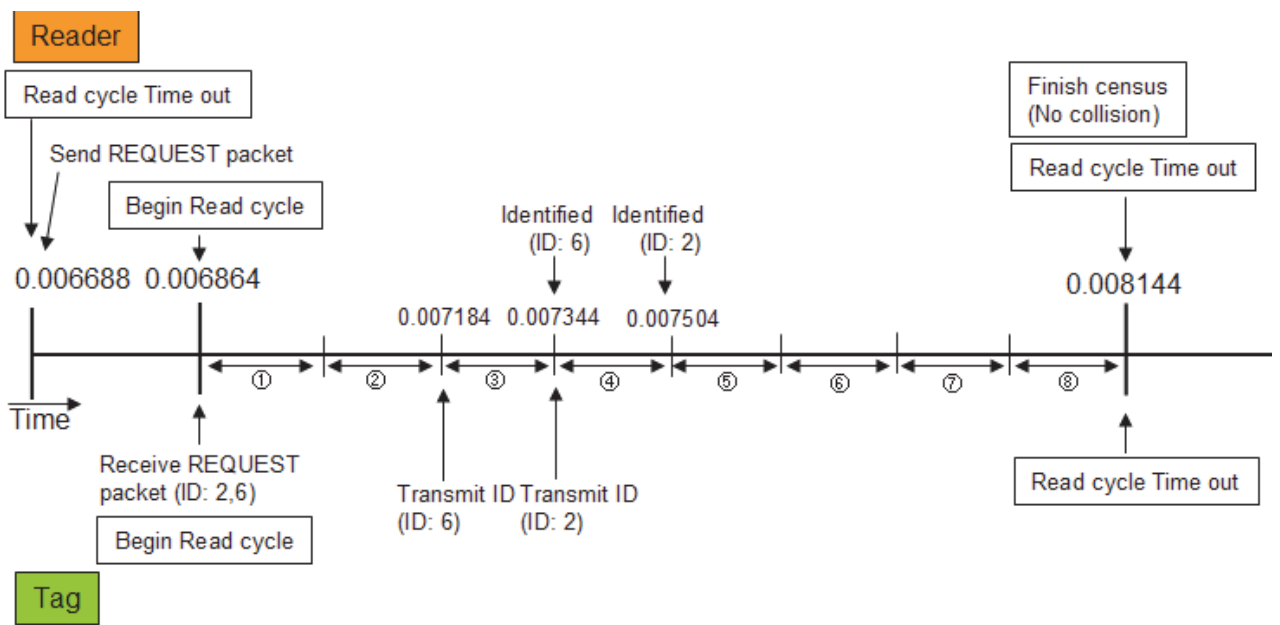

(e) Fifth Read Cycle of the Simulation

Fig. 16. BFSA-Muting Simulation Log

As we can see from Figure 16 (a), when the census begins the reader broadcasts a REQUEST packet to all tags. The transmission delay of a REQUEST packet is 0.000176 seconds since the size of the packet is 88 bits while the data rate is 500,000 bps. We assume propagation and calculation delay are negligible, since events are generated at slot boundaries and propagation delay and computation time will not have an effect on census delay and throughput. As soon as tags receive the REQUEST packet they start their timer to synchronize the read cycle between the reader and tags. Tags can select only one of the slots in the read cycle randomly and transmit a RESPONSE packet which contains tag's ID and CRC to the reader by occupying a single slot, e.g. as we see from Figure 16 (a) each tag send its ID only once in a read cycle based on the definition of the FSA protocol. There are eight slots in a frame in this simulation. And we can see every slot durations in the read cycle is identical. The delay for the transmitting of the RESPONSE packet is the definition of the slot duration. As you see at Figure 13 (b) the size of RESPONSE packet is 80 bits while data rate is $500,000 \mathrm{bps}$. That makes the transmission time of the REQUEST packet to 0.00016 seconds. When multiple tags transmit their ID to the reader with the same slot it causes a collision then the reader can't identify tag's ID successfully. Two collisions occur in the first read cycle, see Figure 16 (a). Three tags (IDs: 1, 2, and 6) transmits their ID by occupying the second slot and two tags (IDs: 3 and 7) are also transmitting during the third slot. Both of them collide and are being discarded. However, a single tag transmission without collision is identified by the reader successfully as can be seen from the fourth, sixth and seventh slot. The first, fifth and eighth slots are idle slots in the first read cycle (frame). When a read cycle (frame) is finished tags can't transmit their ID until the next read cycle begins and the number of identified tags, collided slots, and idle slots are computed and stored by the reader. If there is no collision during a read cycle the census will be completed.

SELECT packets are transmitted together with the tag's ID identified by the reader as soon as a read cycle has completed (as shown in Figure 16 (b)). The purpose of sending SELECT packet is to mute the already identified tags, i.e. forcing them to stop transmitting their IDs. This reduces collisions. 
Three SELECT packets are transmitted as shown in Figure 16 (b) with a tag's ID identified in the previous read cycle. The size of the SELECT packet is 72 bits and because of the data rate being 500,000 bps the transmission delay will be 0.000144 seconds. After transmitting SELECT packets the REQUEST packet is broadcasted to all tags. However, selected tags will disregard this message. Only unread tags will response to the REQUEST packet.

When the REQUEST packet is delivered to all tags the read cycle is started again. The reader can synchronize the start time of the read cycle with tags since reader can calculate the transmission delay of SELECT and REQUEST packet with packets size and data rate. Once the read cycle is started, the procedure of transmit tag's ID, of detecting collision, and of identifying tag's ID is same with ones in the previous read cycle. When the reader detects no collision during a read cycle the census will be finished shown in Figure 16 (e).

\subsubsection{BFSA-non-muting}

There are two major differences from BFSA-Muting; identified tags are not muted and the assurance level is used for finishing the census. For measuring the assurance level after finishing every read cycle and finishing the census successfully, the line 9 of Figure 15 would be replaced with Figure 17.

1 measure current assurance level

2 if (Given Assurance level $<=$ Current Assurance level) then

Fig. 17. Computing Assurance Level of BFSA-Non-Muting

In the BFSA-Non-Muting, tags are not muted at all. Thus, the probability of collision occurrence is higher than the BFSA-Muting and SELECT packet is not necessary to be transmitted to tags.

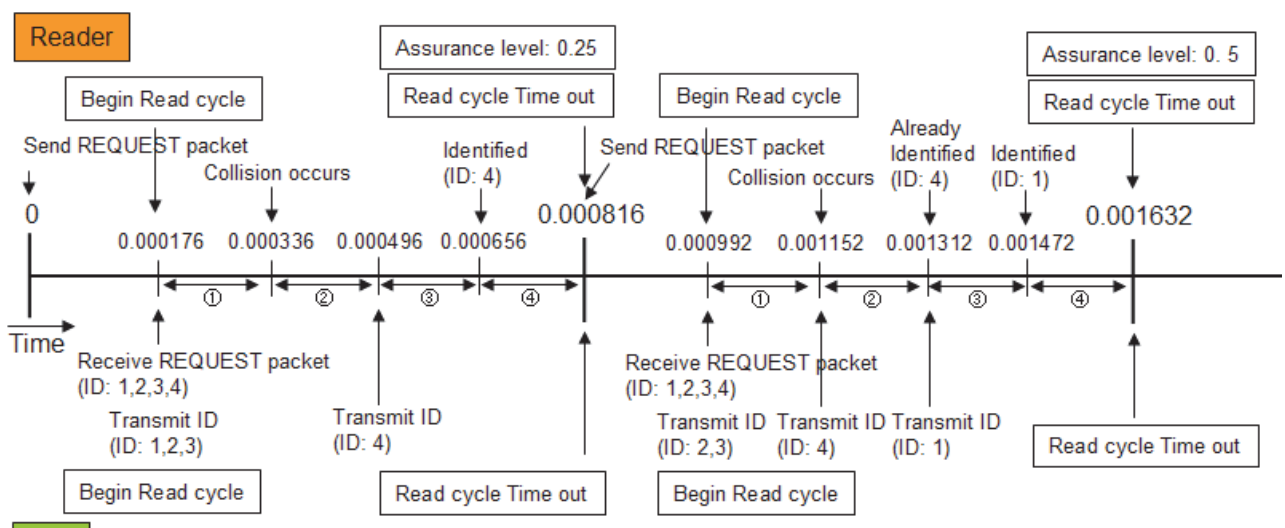

\section{Tag}

(a) First and Second Read Cycle of the Simulation 


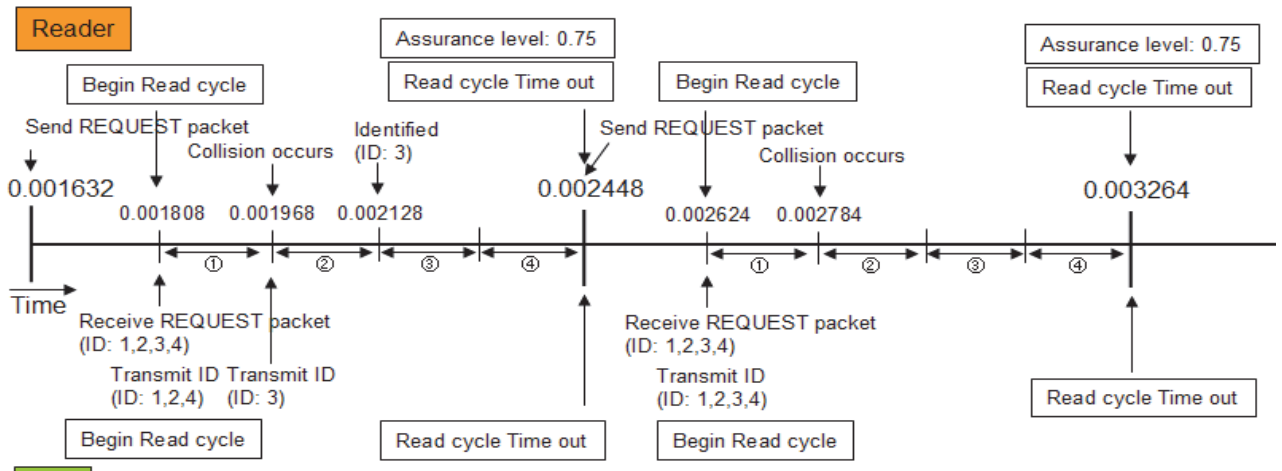

Tag

(b) Third and Fourth Read Cycle of the Simulation

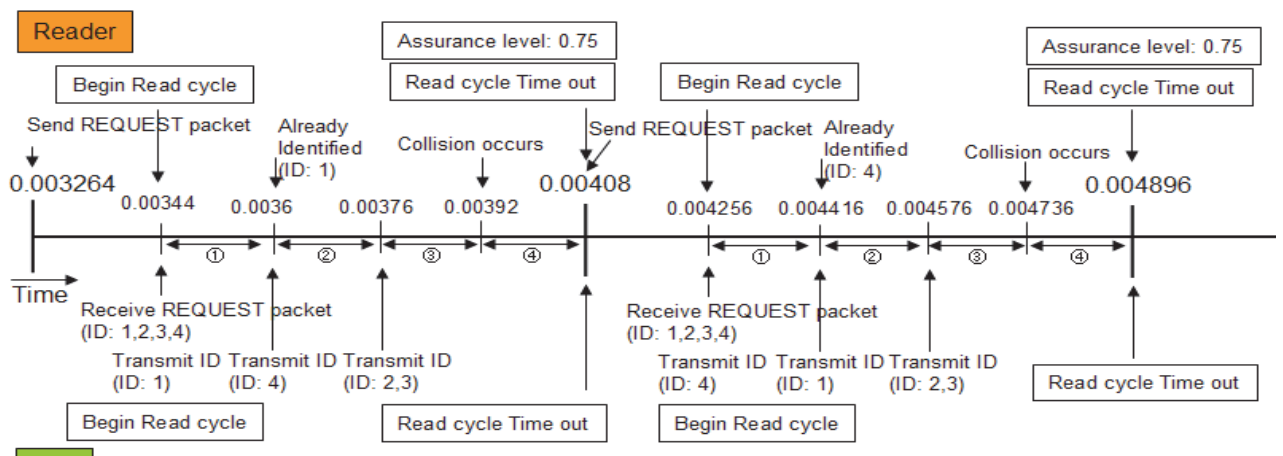

Tag

(c) Fifth and Sixth Read Cycle of the Simulation

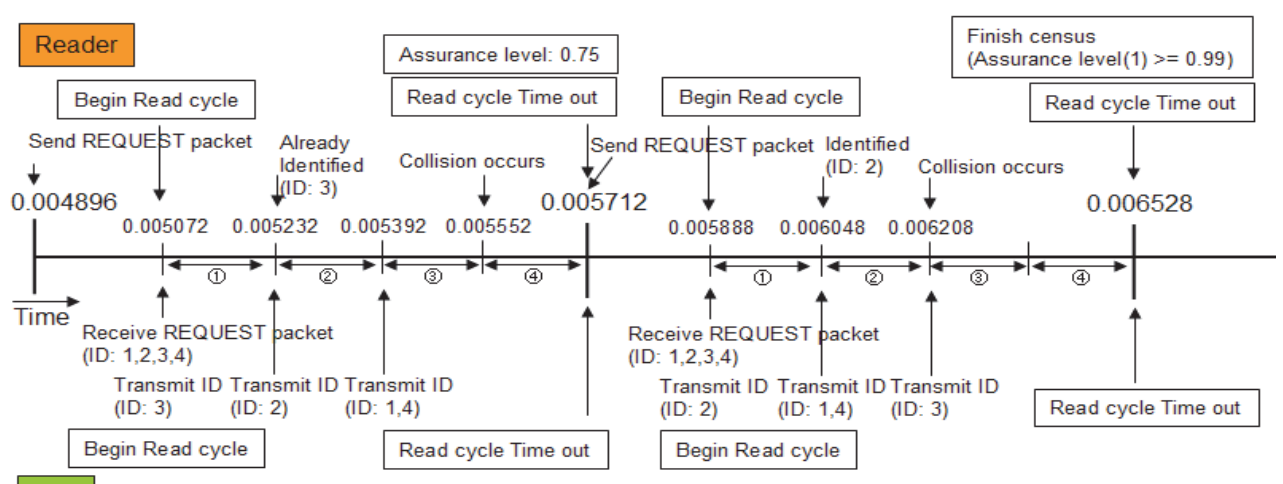

Tag

(d) Seventh and Eight Read Cycle of the Simulation

Fig. 18. BFSA-Non-Muting Simulation Log 
In BFSA-Non-Muting when the census begins the reader transmits REQUEST packet to all tags and they start transmitting their IDs (once in a read cycle). Tags are never muted, so that all tags continue to transmit for the duration of a census, once every read cycle. Another difference between BFSA-Non-Muting and BFSA-Muting is that they have different behavior at the end of each read cycle. As can be seen from Figure 18, the assurance level is measured at the end of every read cycle and is changed according to the total number of identified tags given the total number of actual tags. As shown in Figure 18 (a) the identified tag (ID: 4) sends its ID again during the next frame. Census completes when the assurance level is satisfied, as shown in Figure 18 (d).

\section{Evaluation}

In this Section, we evaluate two parameters: total census delay and network throughput. We compare our simulation results with analytical results, computed by using the equations from Section 3.

\subsection{Total census delay}

Total census delay varies depending on the frame size and the number of actual tags in the BFSA model. If a frame size is either too big or too small as compared to the total number of tags the delay will be longer because of the increased number of idle slots and collision slots respectively, i.e. there is an optimal frame size resulting in the least total census delay for given number of tags. Thus, we first measure the optimal frame size to find the minimal total census delay for given fixed number of tags to be read (identified). Simulation runs were conducted by varying the initial number of tags from 10 to 100 with step of 5 while the given static frame size varies from 10 to 120 with step of 5.10 census procedures were simulated for each frame size and given a specific number of tags.

The minimal total census delay for given static number of tags is shown in Figure 19. Triangle line represents the analytical result of BFSA-Non-Muting, ' $x$ ' line represents simulation result of BFSA-Non-Muting, square line represents analytical result of BFSAMuting, and ' + ' line represents simulation result of BFSA-Muting. For computing the analytical result of BFSA-Non-Muting and BFSA-Muting a computing program was developed [appendix C] and equation 6, 7, 9, 10, 11, 14, 15, 16, 17, 18 and 21 in Section 3 are used.

The minimum total census delay was increased linearly with the number of tags and 100 tag set was identified within $0.25 \mathrm{sec}$ using BFSA-Non-Muting with assurance level 0.99 and 500 Kbps data rate. BFSA-muting took less than $0.1 \mathrm{sec}$ with the same given conditions with BFSA-Non-Muting. The simulation result of BFSA-Muting shows approximately $70 \%$ shorter minimum total census delay than BFSA-Non-Muting simulation result. Both BFSA simulation results show about less than $15 \%$ shorter minimum total census delay than its analytical results in the experiment.

The optimal frame size is acquired from the simulation being used for computing the minimum total census delay in Figure 19. The symbols in Figure 20 are identical with Figure 19. Figure 20 shows us good agreement between the simulation result and the analytical result. The optimal frame size was increased linearly with the number of tags and BFSAMuting has smaller optimal frame size than the one BFSA-Non-Muting has. 


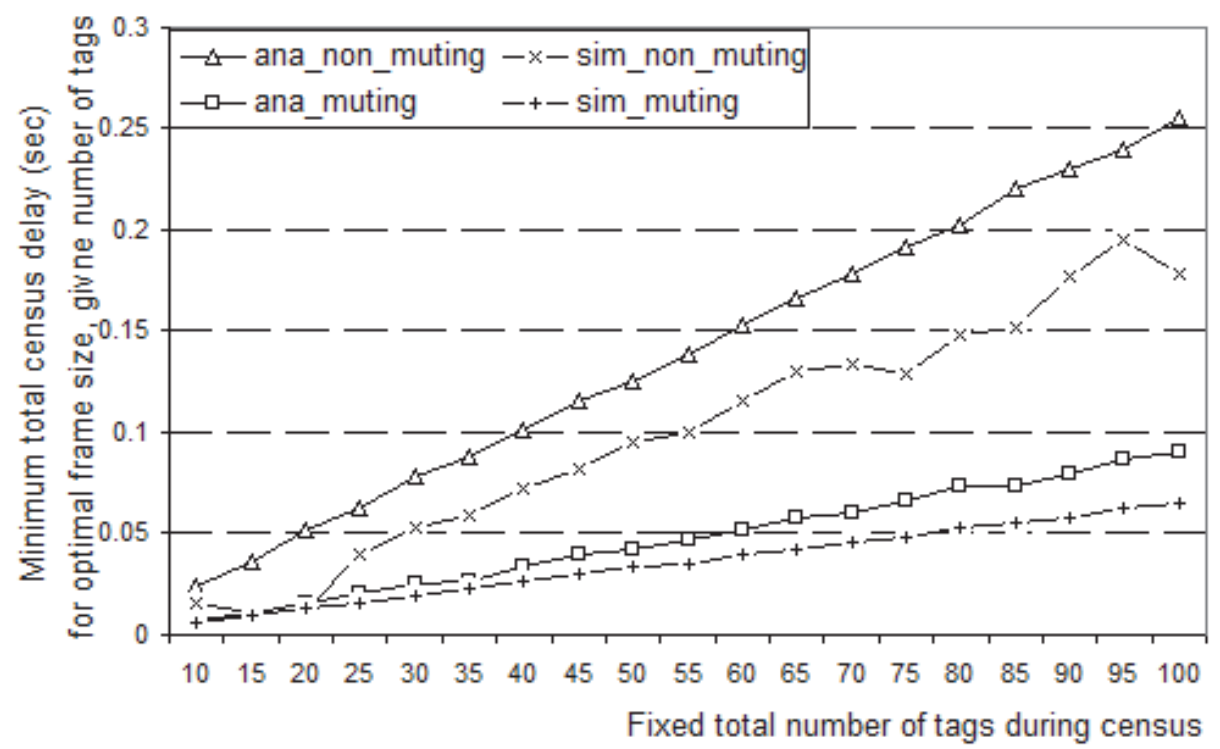

Fig. 19. Minimum Total Census Delay for Given Number of Tags

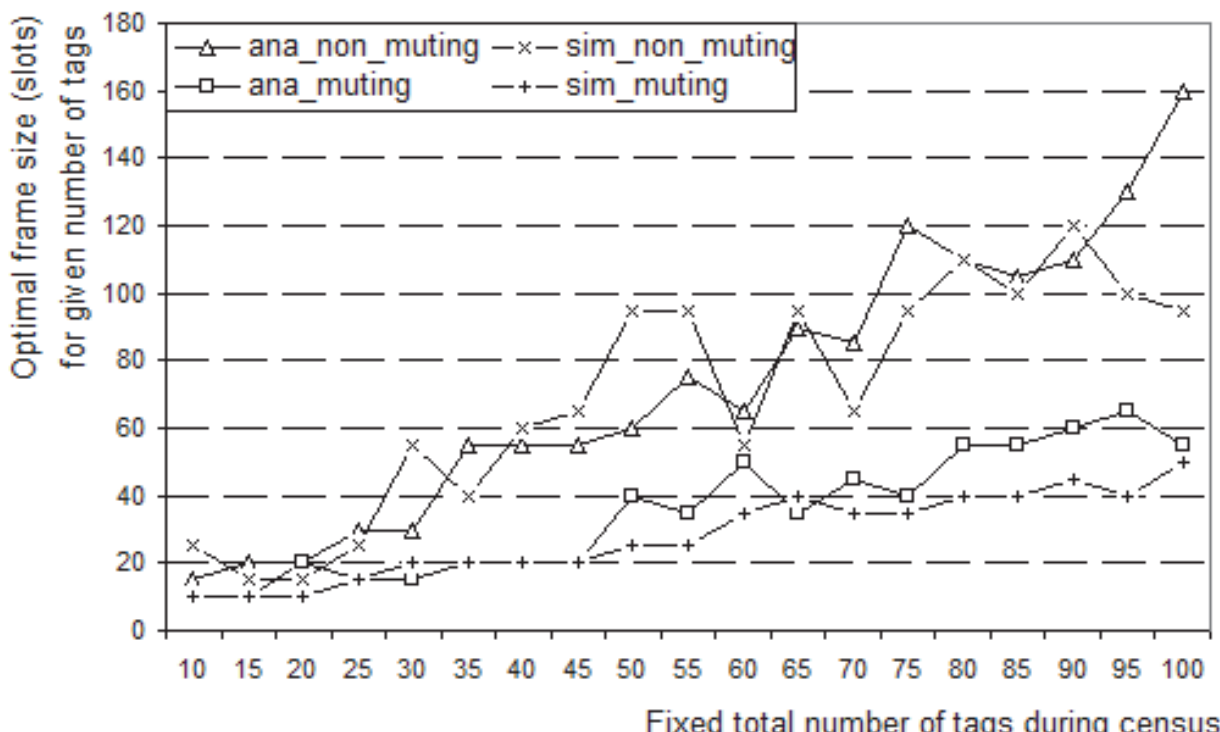

Fig. 20. Optimum Frame Size for Given Number of Tags 


\subsection{Network throughput}

We evaluate three types of network throughput: maximum throughput, minimum throughput, and mean throughput. Network throughput represents the ratio between successfully transmitted number of packets and total number of transmitted packets during census. All of them show good agreement between analytical result and simulation result and they are shown in Figure 21.

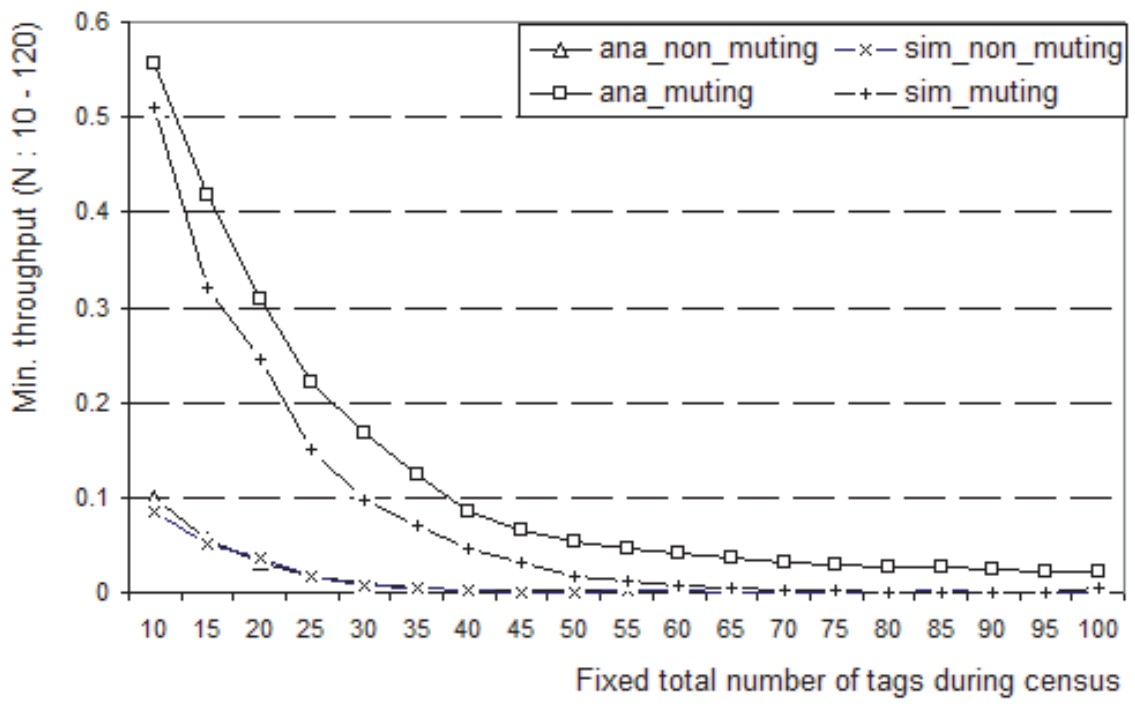

(a) Minimum Network Throughput 


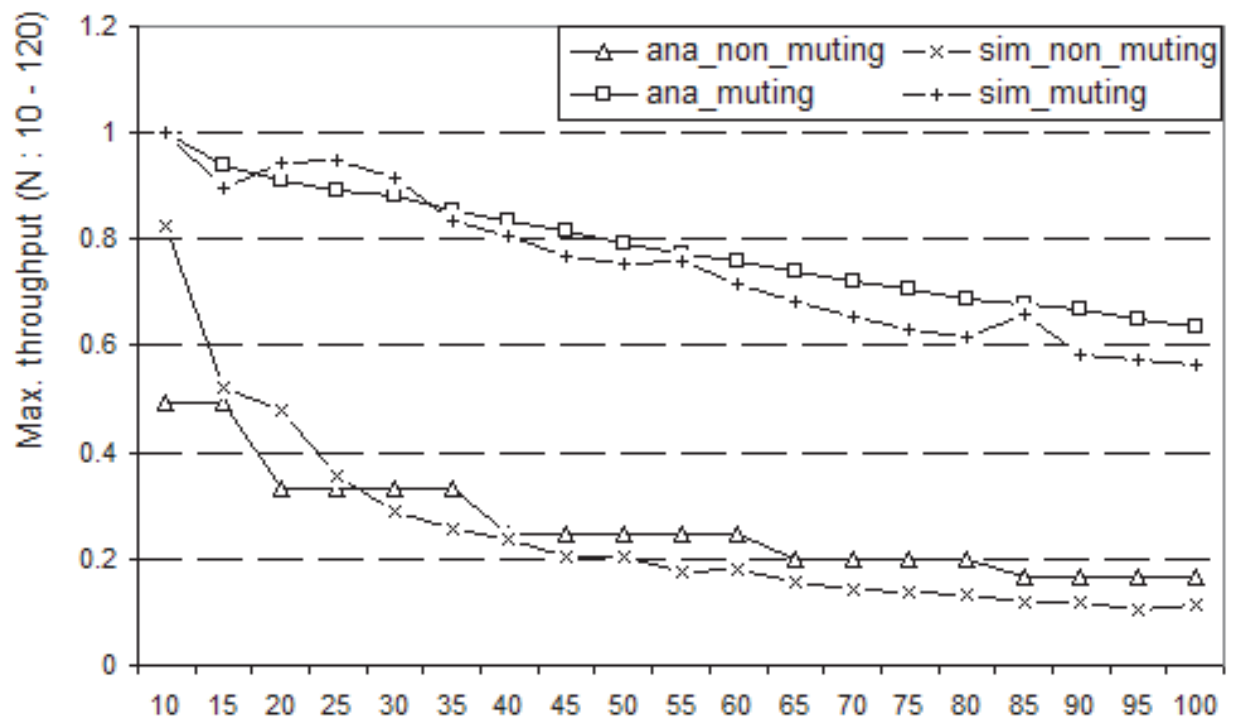

Fixed total number of tags during census

(b) Maximum Network Throughput

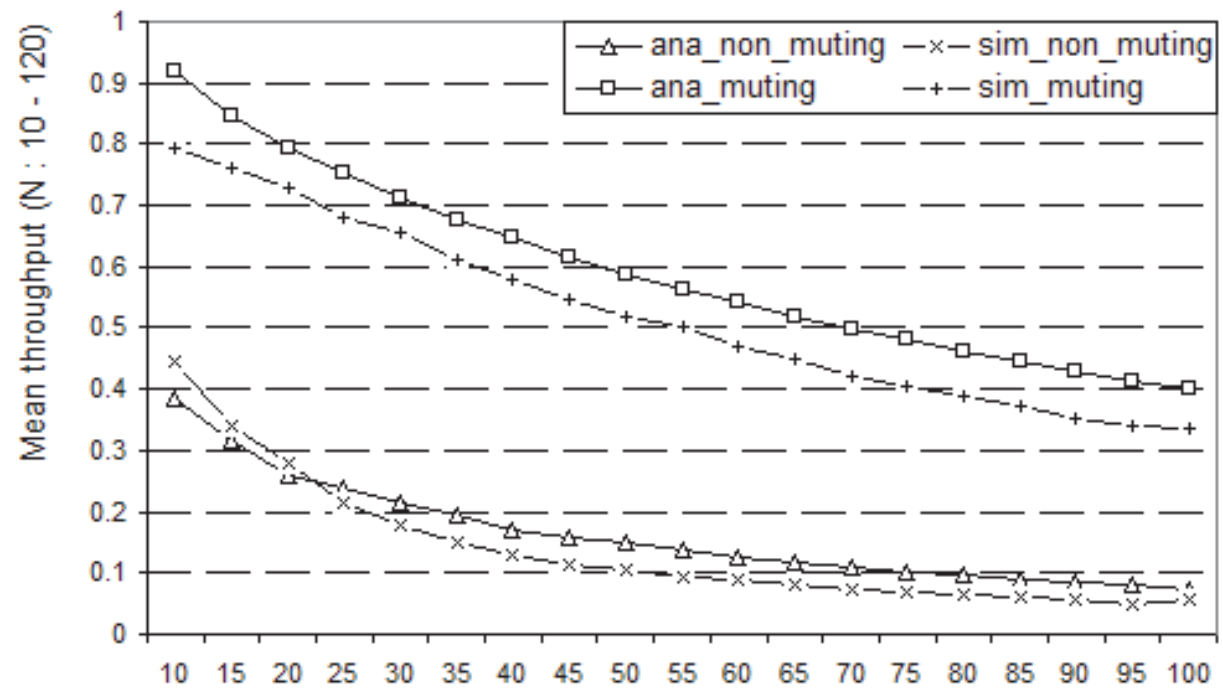

Fixed total number of tags during census

(c) Mean Network Throughput

Fig. 21. Network Throughput 
In Figure 21, network throughput shows good agreement between analytical and simulation result and simulation throughput shows slightly lower than analytical one. Figure 21 tell us that the network throughput of the two BFSA models is getting lower according to the increment of the fixed total number of tags. In Figure 21 (c), we can see mean network throughput of BFSA-muting is $200-400 \%$ greater than the throughput of BFSA-NonMuting. Since in the BFSA-Muting the identified tags keep silent thus the total number of transmitted packet would be reduced while in the BFSA-Non-Muting the identified tags never stop transmitting its ID. Thus the difference between two RFID models makes network throughput different.

\section{Conclusion}

To evaluate the performance of RFID protocols we implemented two BFSA models (Muting and Non-muting). We have used the simulation tool, OPNET Modeler 14. The simulation models were validated by analyzing the $\log$ in the validation Section. In addition, we compared the simulation results against analytical results, generated by using the equations presented in Section 3.

In Section 4, we evaluated total census delay and network throughput by comparing simulation and analytical results. Our simulation results show good agreement with analytical results both for total census delay and for network throughput. We also could see the performance difference of the two BFSA models in terms of the total census delay and the network throughput. As expected, BFSA-Muting performed better in terms of both network throughput and total census delay as compared to BFSA-Non-Muting due to reduction in the total number of transmitted packets.

\section{References}

[Finkenzeller03] Finkenzeller, K., “RFID Handbook," 2nd edition, John Wiley \& Sons, 2003.

[Klair04] Klair, D. K., Chin, K. W. and Raad, R., "On the Suitability of Framed Slotted Aloha based RFID Anti-collision Protocols for Use in RFID-Enhanced WSNs," Computer Communications and Networks, Proceedings of 16th International Conference (August, 2007), pp. 583-590.

[Rom90] Rom, R., and Sidi, M., "Multiple Access Protocols/Performance and Analysis," Springer-Verlag, March 15, 1990. pp. 47-77.

[Want06] Want, R., "An Introduction to RFID Technology," IEEE CS and IEEE ComSoc, Pervasive computing, 2006, pp. 25-33.

[Weinstein05] Weinstein, R., "RFID: A Technical Overview and Its Application to the Enterprise," IEEE Computer Society, May 2005, pp. 27-33.

Electronic Sources:

[Bin05]Bin, Z., Mamoru, K. and Masashi, S., "Framed Aloha for Multiple RFID Objects Identification," IEICE Trans. Comm., Vol.E88-B, No.3 March 15, 2005.

[Cappelletti06] Cappelletti, F., Ferrari, G., and Raheli, R., "A Simple Performance Analysis of Multiple Access RFID Networks Based on the Binary Tree Protocol", ISCCSP March 15, 2006. 
[Computerworld07] www.computerworld.com, “Proctor \& Gamble: Wal-Mart RFID Effort Effective,"http://www.computerworld.com/action/article.do?command=viewArt icleBasic\&articleId=284160, February 26, 2007. [OPNET] OPNET Technologies, http://www.opnet.com.

[Vogt02] Vogt, H., "Efficient object identification with passive RFID tags," Inter. Conf. on Pervasive Computing, LNCS, pp.98-113, Springer-Verlag, March 15, 2002.

[Zürich04] Zürich, E., Burdet, L. A., "RFID Multiple Access Methods," Seminar "Smart Environments", August 15, 2004. 


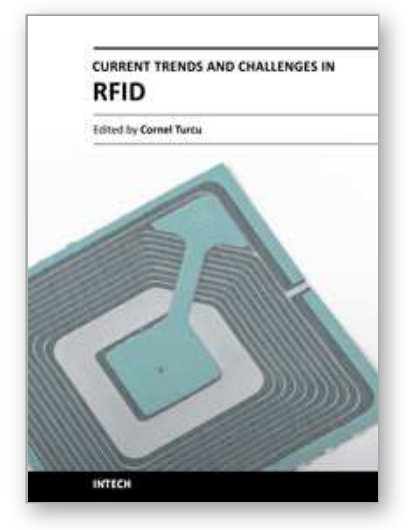

\author{
Current Trends and Challenges in RFID \\ Edited by Prof. Cornel Turcu
}

ISBN 978-953-307-356-9

Hard cover, 502 pages

Publisher InTech

Published online 20, July, 2011

Published in print edition July, 2011

With the increased adoption of RFID (Radio Frequency Identification) across multiple industries, new research opportunities have arisen among many academic and engineering communities who are currently interested in maximizing the practice potential of this technology and in minimizing all its potential risks. Aiming at providing an outstanding survey of recent advances in RFID technology, this book brings together interesting research results and innovative ideas from scholars and researchers worldwide. Current Trends and Challenges in RFID offers important insights into: RF/RFID Background, RFID Tag/Antennas, RFID Readers, RFID Protocols and Algorithms, RFID Applications and Solutions. Comprehensive enough, the present book is invaluable to engineers, scholars, graduate students, industrial and technology insiders, as well as engineering and technology aficionados.

\title{
How to reference
}

In order to correctly reference this scholarly work, feel free to copy and paste the following:

Seungnam Kang and Zornitza Prodanoff (2011). RFID Model for Simulating Framed Slotted ALOHA Based Anti-Collision Protocol for Muti-Tag Identification, Current Trends and Challenges in RFID, Prof. Cornel Turcu (Ed.), ISBN: 978-953-307-356-9, InTech, Available from: http://www.intechopen.com/books/current-trendsand-challenges-in-rfid/rfid-model-for-simulating-framed-slotted-aloha-based-anti-collision-protocol-for-mutitag-identifica

\section{INTECH}

open science | open minds

\section{InTech Europe}

University Campus STeP Ri

Slavka Krautzeka 83/A

51000 Rijeka, Croatia

Phone: +385 (51) 770447

Fax: +385 (51) 686166

www.intechopen.com

\section{InTech China}

Unit 405, Office Block, Hotel Equatorial Shanghai

No.65, Yan An Road (West), Shanghai, 200040, China

中国上海市延安西路65号上海国际贵都大饭店办公楼 405 单元

Phone: +86-21-62489820

Fax: $+86-21-62489821$ 
(C) 2011 The Author(s). Licensee IntechOpen. This chapter is distributed under the terms of the Creative Commons Attribution-NonCommercialShareAlike-3.0 License, which permits use, distribution and reproduction for non-commercial purposes, provided the original is properly cited and derivative works building on this content are distributed under the same license. 